\title{
Optical properties of all-solid microstructured optical fibers
}

\author{
A. Umińska ${ }^{1}$, M. Grabka ${ }^{1}$, S. Pustelny ${ }^{1,2}$, R. Buczyński ${ }^{3}$, I. Kujawa ${ }^{4}$, W. Gawlik ${ }^{1}$ \\ ${ }^{1}$ Department of Photonics, Institute of Physics, Jagiellonian University, Reymonta 4, Kraków, Poland \\ ${ }^{2}$ Department of Physics, University of California at Berkeley, Berkeley, California, USA \\ ${ }^{3}$ Department of Information Optics, Institute of Geophysics, University of Warsaw, Pasteura 7, Warsaw, Poland \\ ${ }^{4}$ Department of Glass, Institute of Electronic Materials Technology, Wolczynska 133, Warsaw, Poland
}

Received October 15, 2012; accepted December 29, 2012; published December 31, 2012

\begin{abstract}
In this Letter, we study optical properties of all-solid microstructured optical fiber made of soft glass. Such fibers have various interesting features including good geometrical stability, low fundamental transmission losses, high Kerr nonlinearity, and large solubility of rare-earth ions. By investigating the transmission mechanism, mode structure, and numerical aperture of the fibers and confronting the results with theoretical calculations we demonstrate that the fibers have optical properties similar to those observed in air-silica microstructured optical fibers. This makes the all-solid soft-glass microstructured fibers very attractive for many potential applications.
\end{abstract}

Unique properties of microstructured optical fibers (MOFs) are the primary reason for their efficient theoretical and experimental investigations. The ability to control fiber absorption, dispersion, mode size and structure allows the fabrication of MOFs with a large mode area [1], high numerical aperture [2], large birefringence [3], extraordinary dispersion values [4], low bend losses [5], and endless single-mode guidance [6]. These features are very attractive for various applications of MOFs in sensing [7], supercontinuum generation [8], telecommunication [9], and industry [10]. While sophisticated structures of MOFs are responsible for unique properties of the fibers, they also make them very sensitive to any fluctuations of waveguide geometry. In fact, one of the major challenges of MOFs is the preservation of optical properties of the fibers along their length. This becomes particularly challenging for air-glass MOFs, as the capillaries of the fiber preform tend to collapse during fiber drawing [11]. In order to prevent such a collapse, pressure is applied during fiber fabrication. Although this technique limits sizable capillary contraction, it does not fully eliminate fluctuations in the fiber geometry, in particular, distortions of air-glass interface, which often introduce light-power losses and alter dispersion of the waveguide [11].

The problem of the instability of optical properties of MOFs may be relaxed by fabrication of all-solid (AS) MOFs [11]. In that case, the geometry deviations are reduced by the application of two or more types of glass with properly matched thermal and chemical properties. A significant difference between refractive indices of glass may provide a sufficient contrast between the core and

http://www.photonics.pl/PLP cladding allowing for the total-internal-reflection (index) guidance or the photonic-bandgap light guidance.

In this Letter, we investigate optical properties of the AS MOF made of two types of soft glass (F2 and NC-21) with the respective refractive indices $n_{\mathrm{F} 2}=1.619$ and $n_{\mathrm{NC}}=1.533$, similar to those investigated in [12]. The fiber possesses hexagonal structure with the lattice constant $\Lambda$ $\approx 2.5 \mu \mathrm{m}$ and $d / \Lambda \approx 0.65$, where $d$ is the diameter of the F2-glass rods (Fig. 1). Its core was formed by replacing seven central F2 rods with a single NC-21 rod and was surrounded by eight rings of the $\mathrm{F} 2$ rods constituting a microstructured cladding of the fiber [Fig. 1(a)]. The fiber has an attenuation of $\sim 13.1 \mathrm{~dB} / \mathrm{m}$ at $855 \mathrm{~nm}$ [12]. All measurements presented in this paper were performed with a fiber of $18 \mathrm{~cm}$ in length.

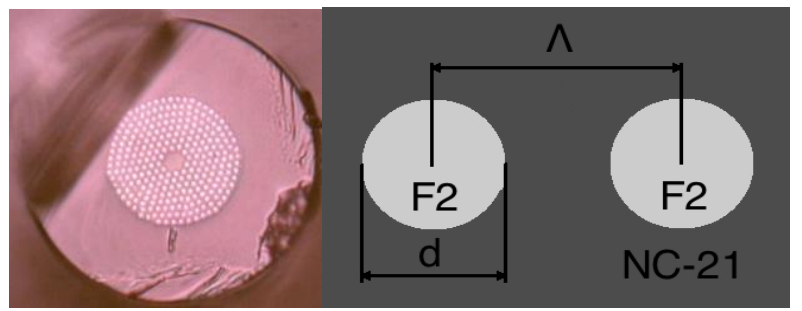

(a)

(b)

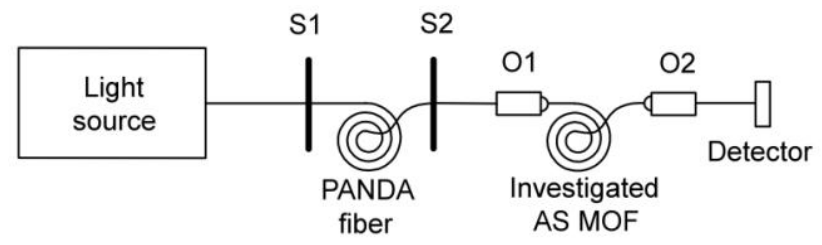

(c)

Fig. 1. (a) Photograph of the AS MOF investigated in this letter. The solid core made of NC-21 glass is surrounded by 8 rings of F2-rods forming the photonic-crystal structure of the fiber. (b) Sketch of the structure. $\Lambda$ denotes the lattice constant and $\mathrm{d}-$ the diameter of the rod. (c) Scheme of the experimental setup. Depending on the investigated property, we used a diode laser operating at $795 \mathrm{~nm}$ (the transverse mode profile and the numerical aperture measurements) or a supercontinuum source (the photonic-bandgap determination). The light was delivered to the investigated MOF trough a polarization-maintaining PANDA fiber. Detector denotes one of three types of detectors: a CCD profiler for the measurements of the transverse mode profile, a power meter placed on a goniometer table for the numerical aperture measurements, and a spectrometer for the photonic band gap study. S1, S2 are the coupling lenses and $\mathrm{O} 1, \mathrm{O} 2$ the microscope objective lenses (for measurements of the numerical aperture there was no decoupling (O2) microscope objective).

(C) 2012 Photonics Society of Poland 
The photonic-bandgap of the fiber was determined using the experimental setup shown in Fig. 1.

A commercial supercontinuum light source (Koheras SuperK Compact of NTK Photonics) emitted light within the 500-2400nm spectral range that was coupled into the fiber with a 40x microscope objective. After the fiber, the light beam was directed to the spectrometer (Red Tide Ocean Optics) operating in the 380-950nm range with a spectral resolution of $2 \mathrm{~nm}$.

The lower curves in Fig. 2 present the spectrum of light transmitted through the fiber (left vertical axis). The data clearly show the existence of two bandgaps, one at $\sim 580 \mathrm{~nm}, 20 \mathrm{~nm}$ wide, and the other much wider $(80 \mathrm{~nm})$ at $\sim 800 \mathrm{~nm}$. The observed broadband background in the spectrum originates from the light propagating in the fiber cladding convoluted with the spectrometer's sensitivity.

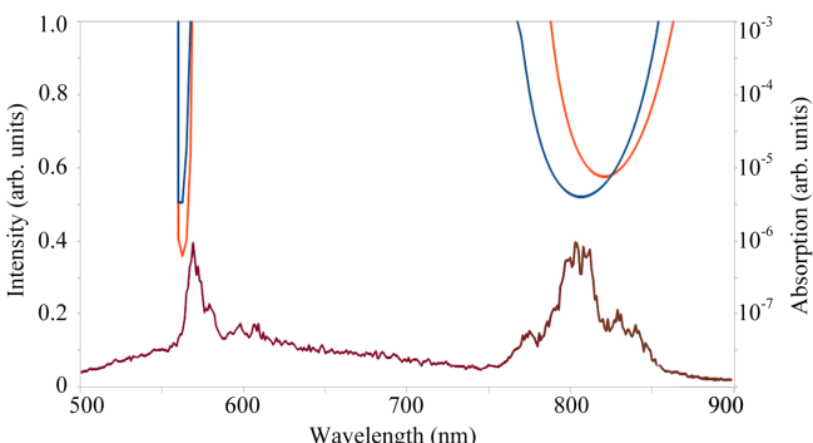

Fig. 3. Transmission spectrum of the fiber. The data reveals the existence of two bandgaps at $580 \mathrm{~nm}$ and $800 \mathrm{~nm}$ and a broadband background. The background arises from not-fully-radiated cladding modes and is determined by a convolution light spectrum and spectrometer spectral sensitivity. The red and blue curves correspond to the absorption coefficients of the two polarization components of the fundamenta mode. Due to some asymmetry of the structure the modes are not fully degenerate.

For the interpretation of the results shown in Fig. 2, the vectorial Maxwell's equations were solved in the fiber structure using a finite element method (FEM) with the ComSol Multiphysics. Scanning-electron-microscope images of the fiber face were used to create a reliable numerical model of the waveguide. The glass dispersion was described with the Sellmeier equations. To theoretically predict the positions of the photonicbandgaps, the transverse modes of the fiber were calculated in a broad range of wavelengths $(500-1000 \mathrm{~nm}$ with $2.5 \mathrm{~nm}$ step). For each wavelength several modes were found. Their shapes and propagation constants were used to verify whether they were photonic-bandgap modes; the modes confined in the core with real part of the propagation constant smaller than the refractive index of the core were chosen. The imaginary part of the propagation constants of such modes allowed determination of the photonic-bandgap positions.

The upper curves in Fig. 2 (right vertical axis) depict the calculated absorption coefficients of the first two transverse modes (Fig. 3). As shown, the simulations revealed two bandgaps in the investigated wavelength range. The positions of the bandgaps coincide with the ones determined experimentally but their widths are slightly different. Such a discrepancy between simulations and the experiment may be expected as the determination of the bandgaps requires a very good geometrical model of the fiber structure as even small changes of the model may introduce significant shifts of the bandgaps. Moreover, the simulations show that the positions of the bandgaps for the two polarization modes (the red and blue upper curves in Fig. 2) are different. This demonstrates the deviation of the real structure from the perfect geometrical structure since a structure with multi-fold symmetry should not possess any birefringence and dichroism [13].

Another important property of an optical fiber is its transverse-mode profile. In order to investigate this property, linearly polarized light emitted from a single mode diode laser operating at $795 \mathrm{~nm}$ was coupled into the fiber. After the fiber, the light was projected onto the CCD beam profiler (for more information on investigating the beam profile and its diffraction pattern evolution see Ref. [14]). Figure 3(a) presents a measured profile of light departing the fiber. As shown, the spatial distribution of the light reveals a hexagonal shape of the core and, to the first order, reproduces a fundamental mode of the fiber [Fig. 3(b)]. A small amount of light present in the first and second rings of the cladding rods proves that higher order modes were also induced in the fibers [Fig. 3(b)]. Some of them have no photonic bandgap and their energy leaks out during propagation. The measurable amount of light in the cladding proves that the fiber was too short to fully radiate the light and the small asymmetry of the measured

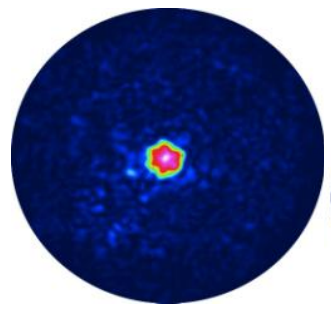

(a)

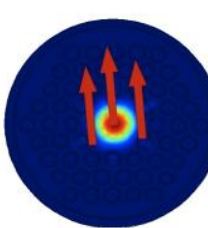

(b)

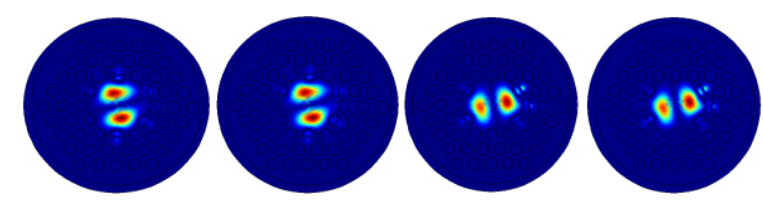

(c)

Fig. 2. Experimentally determined intensity distribution in the fiber (a) and the polarization components of two modes LP01 (b) and LP11 (c). The polarization components of the modes are degenerated: they have the same propagation constant and energy distribution. The colors indicate intensity of the measured/simulated light and the arrows indicate the light polarization (for clarity of the figure the polarization arrows are presented only for the fundamental mode LP01). Polarization vectors were omitted at modes presented in (c) for better clarity. 
profile demonstrates the deviation of the real structure from its perfect geometrical model.

Finally, the numerical aperture of the fiber was measured. For the measurements, the fiber end was installed on a goniometric table with a small-active-area $\left(1 \mathrm{~mm}^{2}\right)$ detector mounted behind the fiber. The intensity of the light was recorded as a function of the goniometric angle (Fig. 4). The data were fit with the Gaussian profile and the numerical aperture of $0.36(1)$ was determined at the level of $5 \%$. At the same time the theoretical considerations based on Ref. [15] predicted NA equal to $0.446(62)$. It is believed that the discrepancy is caused by the diffusion of two types of glass and the continues rather than step change of the refractive index at the interface between fiber main material and rods. Moreover, in a multimode fiber, the transverse modes reveal different propagation constants and hence numerical apertures. Consequently, the observed diffraction comes not only from the fundamental mode but also from higher-order modes (including cladding modes that were not entirely attenuated). This hypothesis might be supported by the fact that the data shown in Fig. 4 deviate from a simple Gaussian profile.

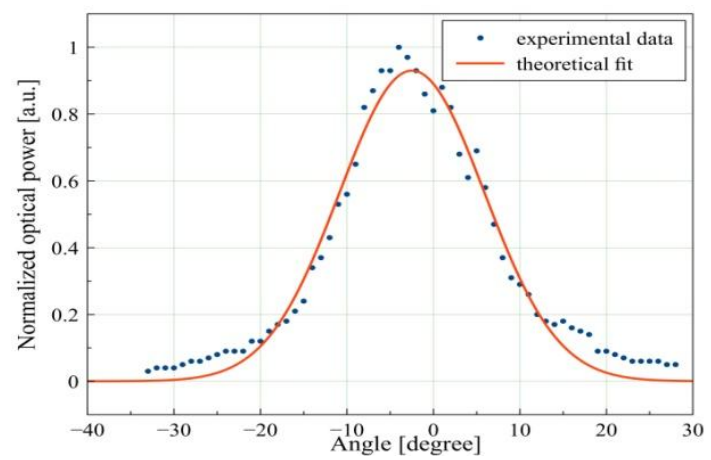

Fig. 4. The measurement of the numerical aperture.

In this Letter, the optical properties of the all-solid microstructured optical fiber made of two types of soft glass were investigated. The results demonstrated the existence of two photonic-bandgaps in the range of 500$1000 \mathrm{~nm}$. The positions of the bandgaps were in good agreement with numerical simulations. The spatial profile of light traversing through the fiber was also investigated. The comparison of experimental data with numerical results revealed that not only fundamental but also higherorder modes are induced in the fiber. This hypothesis was supported by the data collected during the measurements of the fiber numerical aperture. Our results showed that many properties of all-solid optical fibers are identical to those of air-glass fibers with either a solid or hollow core. At the same time, the all-solid fibers made of soft glass are superior over other groups of optical fibers due to their better structural stability and hence smaller fluctuations of their parameters. The important characteristics of the soft-glass fibers are significantly higher Kerr nonlinearity, rare-earth ion solubility, and different transmission characteristics than in conventional (air-silica) microstructured fibers. All these features make the all-solid microstructured fibers very appealing for such applications as mid-infrared spectroscopy, LIDAR, high-gain fiber amplifiers [16]

The research was carried out with the equipment purchased thanks to the financial support of the European Regional Development Fund in the Framework of the Polish Innovation Economy Operational Program (contract no. POIG.02.01.00-12-023/08). It was also supported by the grant of the Polish National Science Centre \# 2011/01/N/ST2/02391 and the Foundation for the Polish Science TEAM Programme. SP is the scholar of the Foundation for Polish Science.

\section{References}

[1] J.R. Hayes, J.C. Flanagan, T.M. Monro, D.J. Richardson, P. Grunewald R. Allott, Opt. Exp. 14(22), 10345-10350 (2006).

[2] N.A. Issa, Appl. Opt. 43(33), 6191-6197 (2004)

[3] M. Antkowiak, R. Kotynski, T. Nasilowski, P. Lesiak, J. Wojcik, W. Urbanczyk, F. Berghmans, H. Thienpont, J. Optics A: Pure Appl. Opt. 7(12), 763 (2005).

[4] W. Reeves, J. Knight, P.St. Russell, P. Roberts, Opt. Expr. 10(14), 609613 (2002)

[5] C. Martelli, J. Canning, B. Gibson, S. Huntington, Opt. Expr. 15(26) 17639-17644 (2007).

[6] T.A. Birks, J.C. Knight, P.St. Russell, Opt. Lett. 22(13), 961-963 (1997)

[7] L. Rindorf, J.B. Jensen, M. Dufva, L.H. Pedersen, P.E. Hoiby, O. Bang, Opt. Expr. 14(18), 8224-8231 (2006)

[8] R. Buczynski, D. Pysz, I. Kujawa, J. Korzeniowski, T. Martynkien. F. Berghmans, H. Thienpont, R. Stepien, Phot. Lett..Poland 1(3), 109 111 (2009)

[9] M. Nielsen, C. Jacobsen, N. Mortensen, J. Folkenberg, H. Simonsen, Opt. Expr. 12(7), 1372-1376 (2004).

[10] W. Wadsworth, R. Percival, G. Bouwmans, J. Knight, P. Russell, Opt. Expr. 11(1), 48-53 (2003).

[11] X. Feng, T.M. Monro, P. Petropoulos, V. Finazzi, D. Hewak, Opt. Expr. 11(18), 2225-2230 (2003)

[12] R. Buczynski, I. Kujawa, R. Kasztelanic, D. Pysz, K. Borzycki, F. Berghmans, H. Thienpond, R. Stepien, Laser Phys. 22(4), 784-790 (2012)

[13] M. Grabka, S. Pustelny, P. Mergo, W. Gawlik, Opt. Expr. 20(13), 13878-13885 (2012)

[14] M. Grabka, B. Wajnchold, S. Pustelny, W. Gawlik, K. Skorupski, P. Mergo, Acta Phys. Pol. A, 118(6), 1127-1132 (2010).

[15] M.J.F. Digonnet, H.K. Kim, G.S. Kino, S. Fan, J. Lightwave Technol. 23(12), 4169-4177 (2005)

[16] H. Ebendorff-Heidepriem, R.G. Moore, T.M. Monro, AIP Conference Proc. 1055(1), 95-98 (2008) 